\title{
Neonatal Encephalopathy and SIADH during RSV Infection
}

\author{
Simonetta Picone, MD ${ }^{1}$ Vito Mondì, MD ${ }^{1} \quad$ Federico Di Palma, MS ${ }^{2} \quad$ Ludovica Martini, MD ${ }^{1}$ \\ Piermichele Paolillo, MD ${ }^{1}$ \\ ${ }^{1}$ Neonatology and Neonatal Intensive Care Unit, Policlinico Casilino \\ General Hospital, Via Casilina, Rome, Italy \\ 2 Medical School La Sapienza University, Piazzale Aldo Moro, Rome, Italy \\ Address for correspondence Simonetta Picone, MD, Neonatology \\ and Neonatal Intensive Care Unit, Policlinico Casilino General \\ Hospital, Via Casilina 1049, 00169 Rome, Italy \\ (e-mail: simpico@libero.it).
}

Am J Perinatol 2019;36(suppl S2):S106-S109.

\begin{abstract}
Objective This report discusses the neurological involvement in respiratory syncytial virus (RSV) infection in neonates.

Study Design We present a case report of a 2-month-old infant affected by a bronchiolitis RSV-positive, with syndrome of inappropriate antidiuretic hormone secretion (SIADH) correlated seizure and encephalopathy.

Results RSV infection can be associated as a serious disease in newborns involving the central nervous system (CNS) and causing seizures or acute encephalopathy. RSV may

\section{Keywords}

- neonatal RSV encephalopathy

- syndrome of inappropriate antidiuretic hormone secretion

- apparent life threatening event

- MRI lesions be also responsible for SIADH and seizures associated with hyponatremia. The RSV related encephalopathy could be caused by different mechanisms, such as direct viral invasion of the CNS or by indirect mechanism mediated by inflammatory cytokines. In addition, it can be favored by severe hyponatremia and SIADH that can cause cerebral edema. Some studies highlight that this virus-related encephalopathy lead to sudden infant death syndrome.

Conclusion In presence of neurological involvement during RSV-infection must be taken in consideration to performing instrumental test to detect cerebral edema. In addiction could be useful to dose inflammatory cytokines, and to consider the immunemodulatory therapy.
\end{abstract}

Respiratory syncytial virus (RSV) infection is the most common cause for admission to the pediatric intensive care unit due to respiratory failure in infancy. In 2015, it was estimated that $22 \%$ of all episodes of severe acute lower respiratory infection in children younger than 5 years of age are associated with RSV. ${ }^{1}$ The virus is also associated with central nervous system (CNS) related symptoms like central apnea, seizures, and encephalopathy. ${ }^{2}$ Central apnea (16-21\% of neurological complications) is common in infants $<2$ months of age and is the most frequent cause of intubation. The central pathogenesis of apnea is not yet completely clear. It could be caused by stimulation of laryngeal chemoreceptor reflex exacerbated by RSV or also by the immaturity of the respiratory center in younger baby, probably mediated indirectly by cytokines. ${ }^{3}$ Febrile seizure during RSV infection are described in children younger than 2 years of age. ${ }^{2}$ It is also possible to have febrile status epilepticus and no febrile seizures in RSV infection (seizures represents $1-6.6 \%$ of the neurological complication). ${ }^{4-6}$ RSV bronchiolitis cause hyponatremia (33\% of cases are isolated hyponatremia). The $4 \%$ of cases have been described hyponatremic convulsions due to syndrome of inappropriate antidiuretic hormone secretion (SIADH), with sodium levels $<123 \mathrm{mEq} / \mathrm{L}$. It is more frequent in infants $\leq 2$ months of age. ${ }^{7}$ Incidence has not changed over the years. The excessive secretion of ADH during RSV infection is related to the degree of air-trapping and hypercapnia (nonosmotic stimuli mechanism). It can also get worse by excessive rehydration to prevent dehydration and by inappropriate use of hypotonic solutions. To prevent cerebral edema because of a slow correction, or osmotic demyelination because of rapid correction, the sodium infusion needs to be $<25 \mathrm{mmol} / \mathrm{L}$ in 48 hours $(0.5 \mathrm{mmol} / \mathrm{h}){ }^{7}$
Copyright (c) 2019 by Thieme Medical Publishers, Inc., 333 Seventh Avenue, New York, NY 10001, USA. Tel: +1(212) 584-4662 ISSN 0735-1631. 
Encephalopathy is characterized by alteration of the state of consciousness and signs of increased intracranial pressure, often associated with seizures, and by the presence of cytotoxic or vasogenic cerebral edema. It is described in 0.1 to $10 \%$ of RSV infections. ${ }^{8,9}$ The pathogenesis is not well known. RSV encephalopathy could be due to a direct action of the virus on the CNS or, more probably, to the para/postinfectious action of cytokines, through an immune-mediated reaction. ${ }^{8,9}$ Only one case with the presence of RSV in the liquor in a 4-month-old infant with febrile convulsions has been described. ${ }^{10}$ In three cases of RSV-associated seizures, very high levels of interleukin 6 (IL-6) in CSF and positivity of the viral genome (RT-LAMP [reverse transcription loopmediated isothermal amplification]) were associated. ${ }^{10}$ Moreover, high levels of inflammatory cytokines in CSF (i.e., IL-6, IL-8, TNF [tumor necrosis factor] $\alpha$ ) has been described in the related VRS encephalopathy. An increased cytokines production may be responsible for cerebral edema, damage to the vascular endothelium, and damage to nerve cells. $^{11,12}$

Several following studies have described different brain magnetic resonance imaging (MRI) findings in RSV encephalopathy: rhombencephalitis, ADEM (Acute Disseminated encephaloMyelitis)-like pattern and limbic encephalitis pattern, ${ }^{9}$ subcortical white matter lesions in temporal, occipital, frontal lobe, ${ }^{4}$ high intensity-lesions in frontal and temporal lobes, ${ }^{10}$ and hippocampus high intensity bilateral lesions. ${ }^{13}$

\section{Case Report}

Bianca had born at 31 weeks of gestational age (GA) by cesarean section because of prelabor rupture of membranes and breech presentation, with birth weight 1,600 g, intubated at 1 minute of life and assisted by conventional mechanical ventilation for 18 hours. One dose of surfactant was administered. No invasive support was necessary by nasal continuous positive airway pressure for 48 hours ( $\max$ Fio2 0.21 ). She was in spontaneous breathing until hospital discharge. No other problems were reported during her stay. She was discharged at
35 weeks of postconceptional age with weight 1,950 g. Cerebral ultrasound was normal until discharge.

During the epidemic season 2017 to 2018, the AIFA (Agenzia Italiana del Farmaco, Italian Medicines Agency) guidelines for RSV prophylaxis included only premature born at $\mathrm{GA}<29$ weeks; therefore Bianca did not receive palivizumab prophylaxis.

At 2 months of age (40 weeks of postconceptional age, weight $3,000 \mathrm{~g}$ ) she was led to the emergency room. She appeared pallid, with peripheral hypoperfusion, generalized hypotonia, hyporesponsiveness, and retroversion of the eyeballs. She also presented with a respiratory pattern of bronchiolitis, started few days before. Her mother reported that while holding Bianca in her arms in full well-being, despite having severe respiratory symptoms for a few days, she became suddenly pale, hyporeactive, hypotonic, "as if she died in her arms"; unapparent life threatening event (ALTE).

The infant was intubated and supported by mechanical ventilation for 10 days. At admission, chest X-ray showed bilateral interstitial disease; subsequently bilateral multiple parailar thickening for bacterial superinfection was evident. C-reactive protein was initially negative, then increased (maximum value: $104 \mathrm{mg} / \mathrm{L}$ ). Pharyngeal swab on admission resulted positive for RSV. The infant presented seizure with tongue fasciculations, rigidity, tonic-clonic muscle contractions of the limbs associated with desaturation, and tachycardia. Seizures were treated with triple antiepileptic therapy (phenobarbital, phenytoin, levetiracetam). At admission, hyponatremia with Na $122 \mathrm{mEq} / \mathrm{L}$ was detected and Bianca was presented with oliguria and compatible with SIADH. Hyponatremia was corrected over the next 48 hours, with sodium infusion around $0.4 \mathrm{mmol} / \mathrm{h}$.

Metabolic tests (ammonia and amino acid blood tests and organic acids in the urine), kidney function, and liver function blood tests were in the normal range. The CSF culture, blood culture, urine culture, gastric aspirate, and rectal swab were sterile.

Bronchoalveolar fluid (BAF) was positive for Klebsiella pneumoniae and Candida albicans.
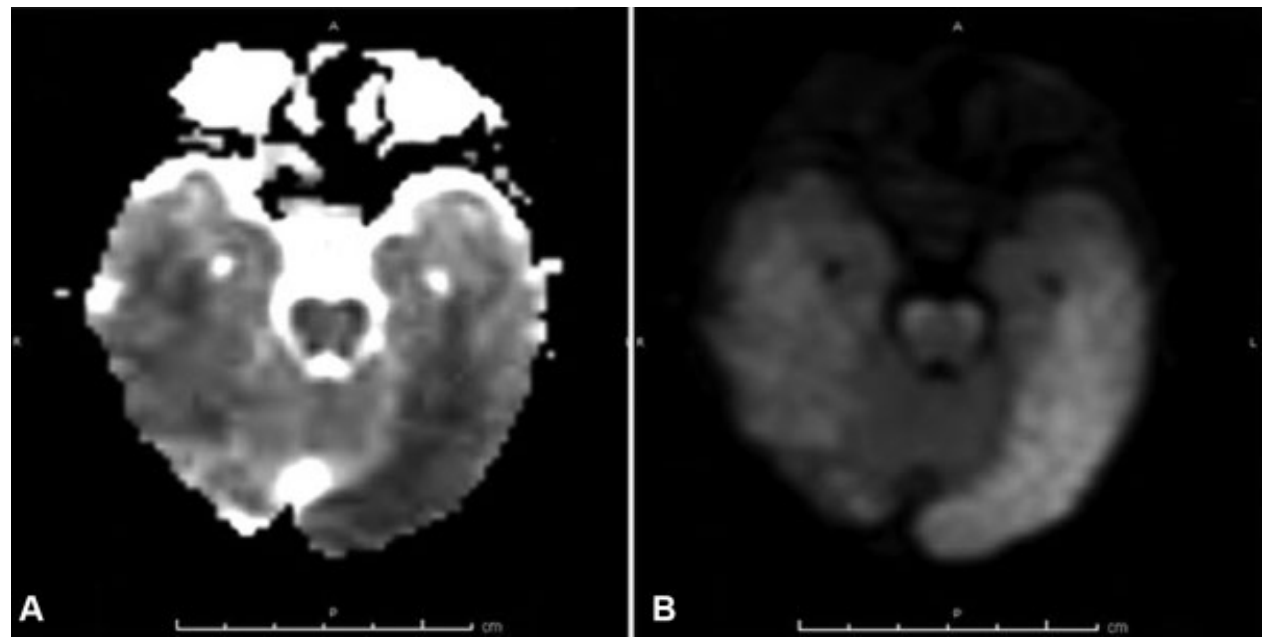

Fig. 1 MRI at 4 days from admission: (A) ADC-MRI, (B) DWI-MRI. Cytotoxic edema of the cortical gray matter particularly in the temporaloccipital lobes on the left. ADC, apparent diffusion coefficient; DWI, diffusion weighted imaging; MRI, magnetic resonance imaging. 


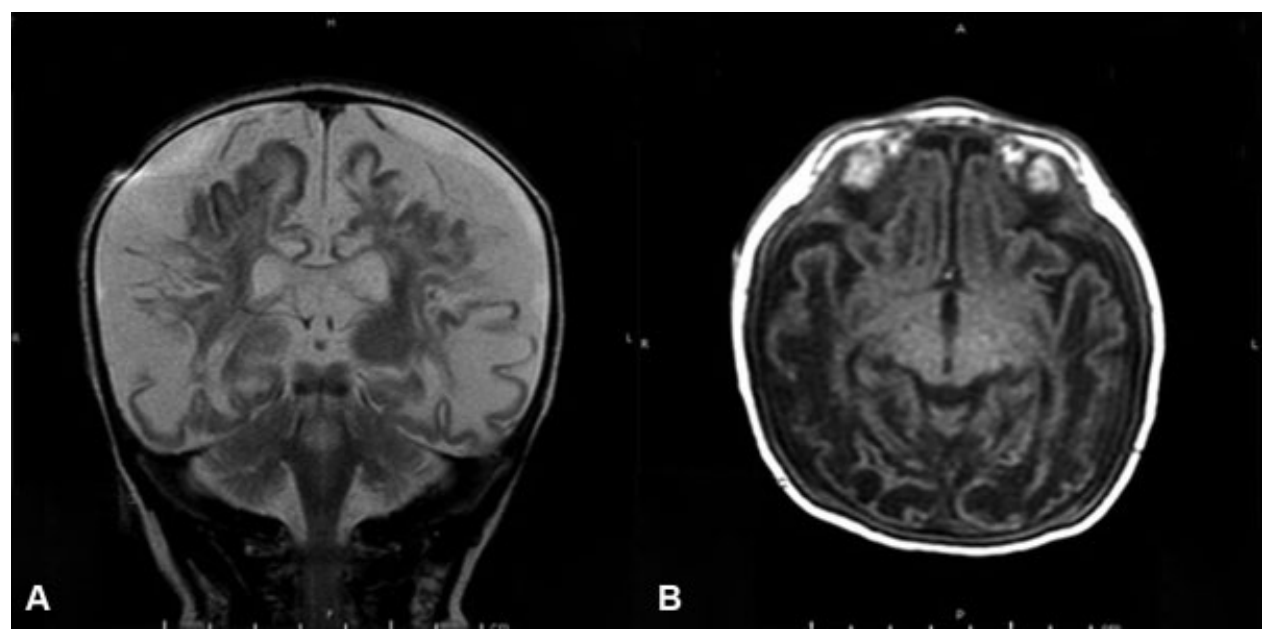

Fig. 2 MRI at 25 days from admission. (A) T2 MRI, (B) T1-MRI. Cystic evolution in the subcortical white matter in the in the temporal- occipital lobes and alteration in the Hippocampi on the right. MRI, magnetic resonance imaging.

PolyCR on BAF was positive for Streptococcus pneumoniae. PolyCR on pharyngeal and rectal swab was positive for RSV A and B. PolyCR on blood was negative for viruses (but RSV was not tested).

PolyCR on liquor was negative for RSV A and B, HSV (herpes simplex virus) 1 and 2, and other viruses and neurotropic bacteria (CMV [cytomegalovirus], Enterovirus A-D, HHV [human herpes virus]6, Parechovirus, VZV [varicella zoster virus], Escherichia coli, Haemophilus influenzae, Listeria monocytogenes, Neisseria meningitidis, Streptococcus agalactiae, S. pneumoniae, Cryptococcus neoformans).

Electroencephalography has showed epileptiform discharges in the frontotemporal regions in both the hemispheres. Cerebral ultrasound was initially normal but approximately 6 days after admission, it showed a pattern of widespread parenchymal hyperchogenicity, and compatibility with diffuse edema.

Four days after admission, brain MRI showed extensive areas of cytotoxic edema of the cortical gray matter, basal and thalamic nuclei, temporal regions, and in particular hippocampi; cytotoxic edema of the subcortical white matter especially at the left temporal lobe (-Fig. 1).

A second MRI after 21 days showed an evolution in cystic lesions of the extensive areas of cytotoxic edema on the subcortical white matter, especially at the level of temporal and parietal lobes bilaterally. No differences were reported in the other areas (-Fig. 2 ).

Bianca was discharged by continuing the antiepileptic therapy with phenobarbital and levetiracetam.

At 1 year of age, Bianca showed impaired neuromotor development, reduction of spontaneous motility, increased muscle tone in the limbs, and axial hypotonia besides reduction of visual functions.

\section{Conclusion}

Our report describes a case of severe encephalopathy in a preterm neonate affected by RSV respiratory infection and not subjected to palivizumab prophylaxis. The newborn presented with a SIADH with hyponatremia associated with oliguria and presented an ALTE; both of these conditions have been associated with RSV infection. The brain radio imaging (RM) showed a severe cytotoxic edema evolved in cystic encephalopathy caused by poor neurological outcome.

Encephalopathy is a rare described complication in RSV infection. Notwithstanding, it is, no frequent, a careful neurological examination need to be performed to detect even the slightest symptoms and signs of neurological involvement. Moreover, in presence of neurological symptoms must be taken in consideration performing all the instrumental tests necessary to detect functional and/or structural abnormalities of the CNS, such as electroencephalography (EEG), cranial computed tomography (CT; urgently), and brain MRI. In addition to the virus detection, the CSF could be useful in dosing the inflammatory cytokines as IL-6, IL-8, and TNF $\alpha$.

Even if a specific therapy for RSV infection is not available, encephalopathy with hyperproduction of inflammatory cytokines and cerebral edema could benefit from immunomodulatory therapy with steroids or immunoglobulins.

\section{Conflict of Interest}

None declared.

\section{References}

1 Shi T, McAllister DA, O'Brien KL, et al; RSV Global Epidemiology Network. Global, regional, and national disease burden estimates of acute lower respiratory infections due to respiratory syncytial virus in young children in 2015: a systematic review and modelling study. Lancet 2017;390(10098):946-958

2 Millichap JJ, Wainwright MS. Neurological complications of respiratory syncytial virus infection: case series and review of literature. J Child Neurol 2009;24(12):1499-1503

3 Erez DL, Yarden-Bilavsky H, Mendelson E, et al. Apnea induced by respiratory syncytial virus infection is not associated with viral invasion of the central nervous system. Pediatr Infect Dis J 2014; 33(08):880-881 
4 Uda K, Kitazawa K. Febrile status epilepticus due to respiratory syncytial virus infection. Pediatr Int (Roma) 2017;59(08): 878-884

5 Sweetman LL, Ng YT, Butler IJ, Bodensteiner JB. Neurologic complications associated with respiratory syncytial virus. Pediatr Neurol 2005;32(05):307-310

6 Antonucci R, Chiappe S, Porcella A, Rosatelli D, Fanos V. Bronchiolitis-associated encephalopathy in critically-ill infants: an underestimated complication? J Matern Fetal Neonatal Med 2010;23(05):431-436

7 Hanna S, Tibby SM, Durward A, Murdoch IA. Incidence of hyponatraemia and hyponatraemic seizures in severe respiratory syncytial virus bronchiolitis. Acta Paediatr 2003;92(04):430-434

8 Zlateva KT, Van Ranst M. Detection of subgroup B respiratory syncytial virus in the cerebrospinal fluid of a patient with respiratory syncytial virus pneumonia. Pediatr Infect Dis J 2004;23(11):1065-1066
9 Park A, Suh SI, Son GR, et al. Respiratory syncytial virus-related encephalitis: magnetic resonance imaging findings with diffusion-weighted study. Neuroradiology 2014;56(02):163-168

10 Kawashima H, Ioi H, Ushio M, Yamanaka G, Matsumoto S, Nakayama T. Cerebrospinal fluid analysis in children with seizures from respiratory syncytial virus infection. Scand J Infect Dis 2009;41(03):228-231

11 Kakimoto Y, Seto Y, Ochiai E, Satoh F, Osawa M. Cytokine elevation in sudden death with respiratory syncytial virus: a case report of 2 children. Pediatrics 2016;138(06):e20161293

12 Kawashima H, Kashiwagi Y, Ioi H, et al. Production of chemokines in respiratory syncytial virus infection with central nervous system manifestations. J Infect Chemother 2012;18(06):827-831

13 Miyamoto K, Fujisawa M, Hozumi H, et al. Systemic inflammatory response syndrome and prolonged hypoperfusion lesions in an infant with respiratory syncytial virus encephalopathy. J Infect Chemother 2013;19(05):978-982 